\title{
Anomalous Decay of Electronically Stabilized Lead Mesas on Ni(111)
}

\author{
Tjeerd R. J. Bollmann, Raoul van Gastel, Harold J. W. Zandvliet, and Bene Poelsema \\ Physics of Interfaces and Nanomaterials, MESA ${ }^{+}$Institute for Nanotechnology, University of Twente, \\ P. O. Box 217, NL-7500AE Enschede, The Netherlands \\ (Received 30 March 2011; published 23 September 2011)
}

\begin{abstract}
With their low surface free energy, lead films tend to wet surfaces. However, quantum size effects (QSE) often lead to islands with distinct preferred heights. We study thin lead films on Ni(111) using low energy electron microscopy and selected area low energy electron diffraction. Indeed, the grown lead mesas show distinct evidence for QSE's. At about $526 \mathrm{~K}$ metastable mesas reshape into hemispheres within milliseconds, driven by a huge reduction in interfacial free energy. The underlying diffusion rate is many orders of magnitude faster than expected for lead on bulk lead.
\end{abstract}

DOI: 10.1103/PhysRevLett.107.136103

The substrate-vacuum, the film-vacuum, and the filmsubstrate interface free energies are three main ingredients governing the equilibrium morphology of a film on a crystalline substrate. Following Young's equation the film wets the substrate when the substrate-vacuum free energy is higher than the sum of the film-vacuum and filmsubstrate free energies. Since their surface free energy is relatively low [1], lead films have an inherent tendency to wet most substrates. Lattice misfit gives rise to an associated stress energy, building up with increasing film thickness. Beyond a critical thickness the film breaks up even if the interfacial energies predict wetting [2]. This feature is considered of lesser importance for lead films since its lattice parameter is much larger than those of most metal substrates, which often leads to higher order commensurability or even incommensurability [3].

A usually disregarded contribution to the total free energy originates from quantum size effects (QSE's). QSE's give rise to specific preferred film heights as the result of a characteristic relationship between the Fermi wavelength, $\lambda_{F}$, and the interlayer distance, $d$ [4-6]. With $d \approx 0.75 \lambda_{F}$, lead is the main representative of this class of materials. Odd layer numbers are preferred initially, later crossing over to even layer numbers [7]. These electronic effects have a profound influence on the film morphology in our low energy electron microscopy (LEEM) and selected area low energy electron diffraction ( $\mu$ LEED) investigation of lead-films on $\mathrm{Ni}(111)$. In addition to QSE's, extremely fast mass transport in ultrathin lead films on $\mathrm{Si}(111)$ has also been reported. Unanticipated fast expansion of lead islands on $\mathrm{Si}(111)$ and anomalous refilling kinetics of laser induced holes have been attributed to fast collective motion inside the wetting layer $[8,9]$. Here we show that ultrafast mass transport is not at all restricted to the wetting layer. Large lead mesas on $\mathrm{Ni}(111)$ decay instantaneously, requiring mass transport rates many orders of magnitude larger than conventional ones for macroscopic lead substrates $[10,11]$.
PACS numbers: 68.55.J-, 68.35.Md, 68.37.Nq, 73.21.Fg

Low energy electron diffraction has been used to monitor the evolution of the wetting layer during deposition of lead on $\mathrm{Ni}(111)$ at $474 \mathrm{~K}$ at a typical rate of about $10^{-3}$ lead atoms per unit cell (uc) per second $(\mathrm{Pb} / \mathrm{uc} / \mathrm{s})$. From $\theta_{\mathrm{Pb} / \mathrm{Ni}} \sim 0.15$ onwards, a faint $(\sqrt{3} \times \sqrt{3})-R 30^{\circ}$ pattern emerges with its maximum intensity around $\theta_{\mathrm{Pb} / \mathrm{Ni}}=0.33$, indicating the formation of a surface alloy [12]. As coverage increases, dealloying and the formation of a lead wetting-layer is observed. An ordered wetting layer covers the entire surface above $\theta_{\mathrm{Pb} / \mathrm{Ni}} \approx 0.40$ and new diffraction peaks emerge at a position corresponding to an in-plane lattice constant of $0.393 \mathrm{~nm}$. From $\theta_{\mathrm{Pb} / \mathrm{Ni}} \approx 0.40$ onwards these $\mathrm{Pb}$ peaks move outward with increasing exposure, indicating compression of the aligned, incommensurable hexagonal lead layer. The coverage in this layer is determined by the combined result of continuously adding new material from the source and the ongoing dealloying. The latter process accelerates with coverage, as is concluded from the increasing shrinkage of the lattice parameter (Fig. 1) until it is completed when the continuous compression ends abruptly at an in-plane lattice constant of $0.350 \mathrm{~nm}$. This observation provides an excellent opportunity for an exact in situ calibration of the deposition rate. We obtain $8 \times 10^{-4} \mathrm{~Pb} / \mathrm{uc} / \mathrm{s}$.

Beyond $\theta_{\mathrm{Pb} / \mathrm{Ni}}=0.510$ the line profiles in Fig. 1 exhibit a surprising peak splitting. It reveals the emergence of two coexisting domains with different in-plane lattice constants: 0.350 and $0.332 \mathrm{~nm}$. These lattice parameters originate from $(7 \times 7)$ and $(4 \times 4)$ moiré patterns. Upon increasing the coverage from $\theta_{\mathrm{Pb} / \mathrm{Ni}} \approx 0.51$ to 0.57 the main spot intensity moves gradually from the less dense $(7 \times 7)$ domains to the denser $(4 \times 4)$ domains (bottom panels Fig. 1). This remarkable structure of the wetting layer requires discussion. The nearest-neighbor distance in bulk lead is $0.350 \mathrm{~nm}$. With the generic tendency for tensile stress at surfaces [13], we suggest that the $(7 \times 7)$ domains involve tensile stress. A slight compression of the $\mathrm{Pb}$ layer is needed to lock in a commensurate $(4 \times 4)$ structure. 

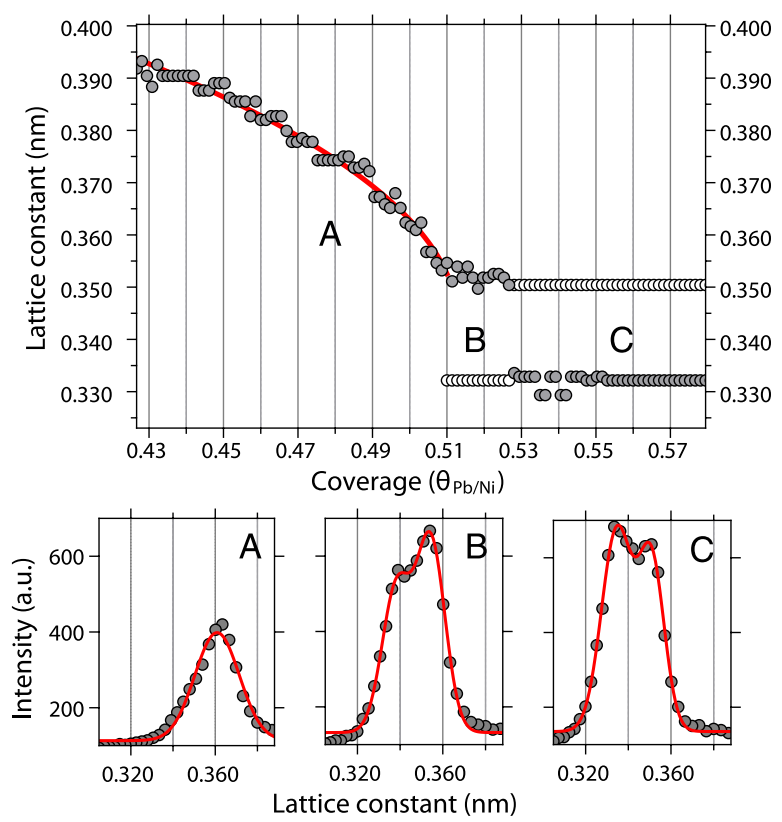

FIG. 1 (color online). LEED data obtained at $35.0 \mathrm{eV}$ during $\mathrm{Pb}$-deposition at $474 \mathrm{~K}$. Upper panel: The in-plane lattice constant, as derived from the position of the $\mathrm{Pb}(10)$ peak as a function of coverage. Open circles denote the lower intensity variant. The solid curve serves to guide the eye. Lower panels: Line profiles of this peak, taken along the $\left(\begin{array}{ll}1 & 0\end{array}\right)$ direction at the coverage indicated by $A, B$, and $C$. The open and closed circles refer to, respectively, the strong and the weak peak.

As a measure of the coordination of an individual $\mathrm{Pb}$ atom we take the sum of the lateral components of its position vector, measured along the directions of the 3 nearest $\mathrm{Ni}$ atoms underneath, relative to those values for an atom in a hollow site. For an on-top, bridge, and threefold hollow site we find (expressed in Ni lattice constants): $1.734,0.444$, and 0 , respectively. The respective average numbers for the $(7 \times 7)$ and the $(4 \times 4)$ structures are 0.550 and 0.618 . This clearly suggests that the coordination is higher and thus the binding stronger for the $(7 \times 7)$ than for the $(4 \times 4)$ structure, in line with the observations. The strong binding to the substrate has a down side in the buildup of compressive lateral stress. The system can only relieve this by keeping the $(4 \times 4)$ patches small and alternating them with low tensile stress $(7 \times 7)$ patches. Differently stressed domains give rise to self-assembly [14]; compare, e.g., $\mathrm{Pb} / \mathrm{Cu}(111)$ [15]. Unfortunately, it is impossible to image these domains. This is due to either the small domain sizes, which are below the spatial resolution of the instrument $(\sim 7 \mathrm{~nm})$ and/or too fast fluctuations of the domain boundaries. The energy cost of domain walls is low due to the alignment to the substrate and similar density, leading indeed to narrow domains and fast fluctuations.

Beyond $\theta_{\mathrm{Pb} / \mathrm{Ni}} \approx 0.55$ lead mesas develop. These mesas are fully relaxed since $\mu$ LEED only shows the bulk lead lattice constant. This is in line with the low misfit energy,

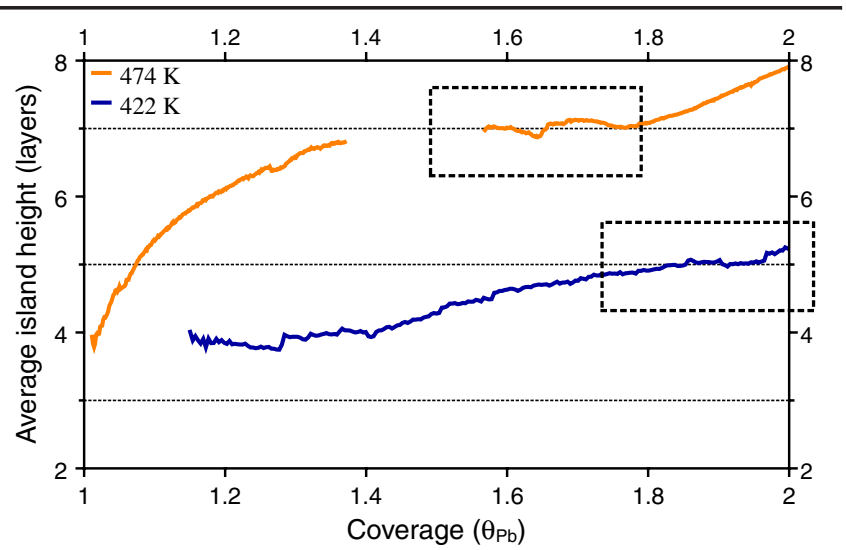

FIG. 2 (color online). The average island height during growth at $422 \mathrm{~K}(\mathrm{FoV}=4 \mu \mathrm{m})$ and $474 \mathrm{~K}(\mathrm{FoV}=10 \mu \mathrm{m})$ as a function of coverage in ML. The dashed boxes indicate situations were the vast majority of the islands attains preferred heights of 5 layers $(422 \mathrm{~K})$ and 7 layers $(474 \mathrm{~K})$. The data were obtained from images with a field of view of $4 \mu \mathrm{m}(422 \mathrm{~K})$ and $10 \mu \mathrm{m}(474 \mathrm{~K})$.

which allows the continuous compression of the wetting layer. From here onwards we define a monolayer (ML) as the equivalent of a bulk $\mathrm{Pb}(111)$ layer. The average height of the growing mesas as a function of the integral coverage, expressed in ML, is shown in Fig. 2 for $422 \mathrm{~K}$ and $474 \mathrm{~K}$. The nucleation phase is finished at about 1.2 and $1 \mathrm{ML}$, respectively. The average height of the mesas growing at $474 \mathrm{~K}$ first increases then flattens out at an island height of 7 layers at a total coverage of 1.5-1.75 ML. At $422 \mathrm{~K}$ the average height flattens out at 5 layers high islands at 1.85-1.95 ML. The flat part of the curves reveals that the islands have preferred heights of 5 and 7 layers, but merely expand in a lateral fashion. Further evidence for a preferred height of 5 layers at $422 \mathrm{~K}$ is given in Ref. [16]. These preferred heights indicate electronic growth for $\mathrm{Pb} / \mathrm{Ni}(111)$. Thus where the interfacial energies clearly favor wetting and no sizeable misfit related stress energies play a role, we find the evolution of a rough growth front with preferred heights, which can only be attributed to QSE's.

The lead mesas most likely nucleate on the domain boundaries or on low density domains since adatom diffusion is slower on patches with tensile strain [17]. The progressively growing islands lock in at these sites, eventually leading to large coalesced lead mesas that anchor at multiple nucleation sites (Fig. 3). Note that $\mu$ LEED shows that the wetting layer maintains its double domain structure even after deposition of more than $10 \mathrm{ML}$ lead. The most eye-catching observation is illustrated in Fig. 3, showing three LEEM images acquired after depositing 12.5 ML of lead on $\mathrm{Ni}(111)$ at $474 \mathrm{~K}$. The left panel shows the surface just after deposition. Subsequently, the sample is heated slowly and the middle and right images have been taken $1 \mathrm{~s}$ apart at $526 \mathrm{~K}$. The grey field represents the wetting layer and the dark features are lead mesas, which cover $31 \%$ of 

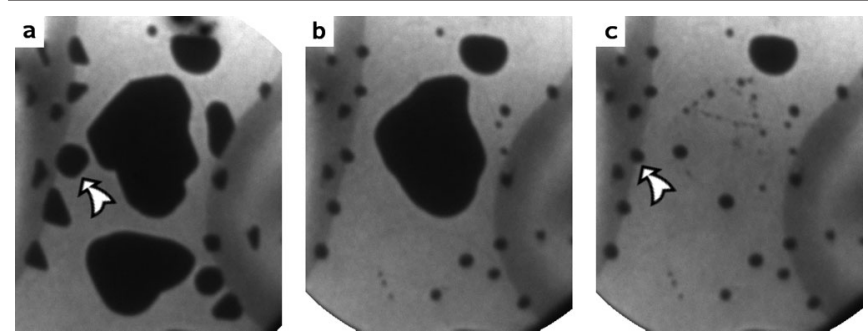

FIG. 3. LEEM images $\left(8.4 \times 10.2 \mu \mathrm{m}^{2}\right)$ taken with $20.0 \mathrm{eV}$ primary electrons after deposition of $12.5 \mathrm{ML}$ of lead at $474 \mathrm{~K}$. The acquisition time for each image is $80 \mathrm{~ms}$. The dark features are lead mesas and the light-gray areas correspond to the wetting layer. The curved features left and right are substrate step bunches. (a) was measured immediately after ceasing the deposition. (b),(c) were measured at $525 \mathrm{~K}$ within a time interval of $1 \mathrm{~s}$, during subsequent slow heating. The white arrows indicate the $\mathrm{Pb}$ island displayed in Fig. 4 (right).

the visible area. The fully relaxed $\mathrm{Pb}$ mesas have an average height of about 38 layers. The central image was recorded at a critical temperature of $525 \mathrm{~K}$. It shows a large central island with a projected area of about $1.14 \times 10^{7} \mathrm{~nm}^{2}$, which is thus composed of about $4.1 \times 10^{9} \mathrm{~Pb}$ atoms. The right hand image was taken just $1 \mathrm{~s}$ later. Within less than the image acquisition time $(80 \mathrm{~ms})$, the huge $\mathrm{Pb}$ mesa collapses into several much smaller ones with an integrated projected area of about $0.6 \times 10^{6} \mathrm{~nm}^{2}$. It thus seems that $3.9 \times 10^{9}$ atoms have disappeared.

This unexpected extremely fast apparent disappearance of more than $10^{9}$ lead atoms needs an explanation. The straightforward options, dissolution into the bulk or desorption into vacuum, both fail: dissolution into the bulk involves Ni bulk vacancy formation; the corresponding adatoms would yield huge step rearrangements, which are not observed [18] and, respectively, remaining compact ad structures persist at $525 \mathrm{~K}$ on a many orders of magnitude larger time scale. Indeed, the Ni bulk vacancy diffusion length is estimated at less than 1 lattice constant within the transition time and the lead vapor pressure at $525 \mathrm{~K}$ is $>10^{6}$ times too low to account for the swift disappearance of more than $10^{9}$ lead atoms. Ostwald ripening is also ruled out since no mass exchange with lead mesas in the vicinity is observed. Therefore, due to conservation of mass, the spectacular lateral shrinkage of the lead mesas must be accompanied by a rapid height increase. This leads to an estimated height of a few hundred nanometers and implies that compact 3D structures emerge $[19,20]$.

Figure 4 provides more insight into the mechanism underlying the sudden transition of the mesas into compact 3D structures. Figure 4 (left) shows a cartoon exemplifying this transition. A circular disk (mesa) is transformed into a hemisphere based in the center. The latter geometry is inspired by the findings for an equilibrium shaped leadparticle on $\mathrm{Cu}(111)$ [20]. This is consistent with image 4 (right), which is a snapshot from the area centered around the lead mesa indicated by the white arrow in Fig. 3(a). Within the first part of the acquisition of this image $(80 \mathrm{~ms})$ the lead island is still a circular lead mesa represented by the larger, lighter gray circle. After the collapse a smaller, darker gray circular feature representing the compact state is seen near the center of the gone mesa. This observation is rare: in less than $5 \%$ of the events we could record the transition as it happened. This implies that the transition time is very short indeed. This is further reiterated by the lack of additional contrast within the larger circle. The transition time, $\tau$, is estimated to be only a few milliseconds.

If we assume that the mesa with a diameter, $2 \times r_{2 \mathrm{D}}$, and a height, $h$, of $10.9 \mathrm{~nm}$ (38 layers) collapses into a hemisphere with radius $r_{3 \mathrm{D}}$ [dark gray circle in Fig. 4 (right)], we can determine the radius of the sphere by using the relation: $r_{3 \mathrm{D}}=(3 h / 2)^{1 / 3} \times r_{2 \mathrm{D}}^{2 / 3}$. For the example in Fig. 4 (right) with $r_{2 \mathrm{D}}=357 \mathrm{~nm}$, one obtains $r_{3 \mathrm{D}}=128 \mathrm{~nm}$, which is consistent with Fig. 4(b). This finding strongly supports the above assignment for the compact lead structure.

This finding is highly significant. First of all, the equilibrium shape of an FCC crystal is a truncated octahedron with (111) and (100) facets. At elevated temperatures, as is the case here (only $12.5 \%$ below the bulk melting temperature), the ridges are rounded and the equilibrium of a

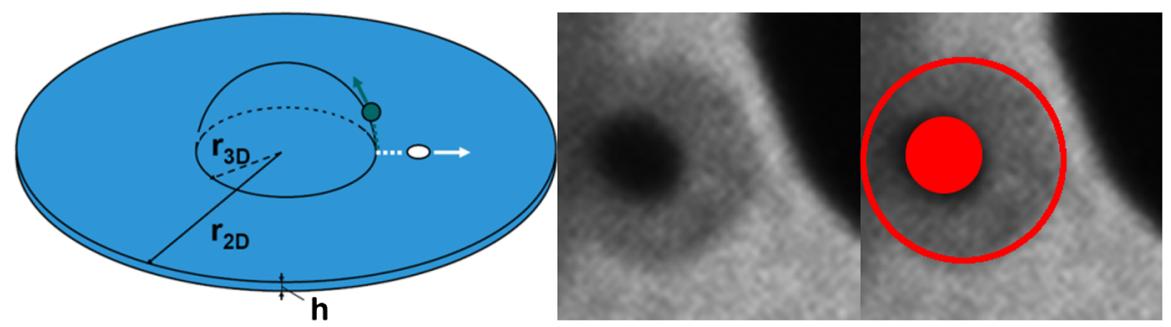

FIG. 4 (color online). (left) Cartoon of the shape transition process. Vacancy-adatom pairs are generated thermally at the contact line between the hemisphere and mesa. The mass transfer takes place via radial motion of vacancies (white) to the outer mesa-rim and of adatoms (green) moving until its accommodation on the hemisphere featuring the compact island. (right) Part of an image ( $1 \mu \mathrm{m}^{2}$ ) at $519 \mathrm{~K}$ during slow heating with the collapse of a $\mathrm{Pb}$ mesa. The $\mathrm{Pb}$ island shown in this figure is indicated by the white arrow in Figs. 3(a) and 3(c). The lighter gray area illustrates the $\mathrm{Pb}$ mesa before its collapse, while the darker central area represents the compact island (see text). The circle and disk represent the shapes described in the text. 
crystallite quite closely resembles a sphere. Second, and more important, a hemisphere implies a contact angle with the substrate of $90^{\circ}$ and therefore at $525 \mathrm{~K}$ the surface free energy of the interface between the wetting layer and vacuum and the free energy of the interface between the $\mathrm{Pb}$ crystallite and the $\mathrm{Ni}(111)$ surface must be (about) identical. Therefore, in considerations of the total interfacial free energy of this system the size of the contact area between the lead crystallites and $\mathrm{Ni}(111)$ can be left out of the equation.

A comparison of Figs. 3(a) and 3(c) shows that nearly all other ad structures underwent a similar shape transition. The large mesa that still persists in the upper region transforms only a few seconds later. Without exception the transitions are almost instantaneous and occur first near preexisting steps. The decay of the lead mesas occurring at about $525 \mathrm{~K}$ is driven by the gain in total free energy that results from the increased volume-to-surface ratio in the transition from mesas to hemispheres. We can now estimate the gain in surface free energy using the example of Fig. 4 (right). Using the measured numbers for $r_{2 \mathrm{D}}$ $(357 \mathrm{~nm})$ and $r_{3 \mathrm{D}}(128 \mathrm{~nm})$ one obtains a reduction of the lead-vacuum interface area of $7.22 \times 10^{5} \mathrm{~nm}^{2}$. Using a surface tension $0.272 \mathrm{~J} / \mathrm{m}^{2}$ [21], one obtains a total gain in free energy of $1.23 \times 10^{6} \mathrm{eV}$. This leads to a reasonable energy gain of $6.3 \mathrm{meV} /$ atom. An analysis of the transition of the large central island into several compact ones leads to a similar estimate.

The ultrafast collapse of the $\mathrm{Pb}$ mesas involves giant mass transport rates. In the example of Fig. 4 about $2 \times 10^{8}$ lead atoms have to assume different positions within milliseconds. For an estimate of the classic mass transfer rate we use the cartoon in Fig. 4 (left). Note that the nearest neighbor distance, $a_{n n}$ for lead is $0.35 \mathrm{~nm}$. As a work hypothesis we assume that the mass transfer takes place via the creation of adatom-vacancy pairs at the mesahemisphere contact line. The vacancies diffuse towards the mesa-rim and the adatoms diffuse to the top of the hemisphere across ascending steps with an associated effective energy barrier $E_{A s}$. The adatom-vacancy pair formation energy is denoted by $E_{V A}$ and the activation barrier for diffusion of vacancies by $E_{D}$. Diffusion is assumed to be strongly biased, resulting in a radial motion of the vacancy from the center towards the outer rim. One then obtains for the transition time $\tau$ with activated hopping:

$$
\tau \approx \frac{38}{\nu_{0}} \pi\left[\left(r_{2 \mathrm{D}} / a_{n n}\right)^{3} / 3\right] \exp \left[\left(E_{D}+E_{V A}+E_{A s}\right) / k T\right]
$$

with $\nu_{0} \approx 10^{13} \mathrm{~s}^{-1} ;\left(r_{3 \mathrm{D}}^{3} \ll r_{2 \mathrm{D}}^{3}\right)$. The prefactor $(0.004 \mathrm{~s})$ is already of the order of the few milliseconds found experimentally for $\tau$, while the effective ascent energy equals $0.26 \mathrm{eV}[5]$ and the activation energy for vacancy diffusion in $\mathrm{Pb}(111), E_{D}$, is lower than that for $\mathrm{Cu}(111)(0.25 \mathrm{eV}$ [21]) and higher than that for lead adatoms on $\mathrm{Pb}(111)$ (80 $\mathrm{meV}$ [22]). The inevitable conclusion is that the traditional hopping processes are unable to explain the extremely fast mass transport during the decay of the giant islands. The same is true for other scenarios we could think of. Some kind of concerted motion in which the lead atoms move collectively towards global positions with a lower free-energy must take place. Unfortunately, the process is too fast to access with currently available techniques.

The still remaining question is why the two-domain structure of the wetting layer disappears at $520-525 \mathrm{~K}$. A conclusive answer cannot be given since no access to the morphological details of the domains is available. However, we stress that the transition of the mesas into hemispheres coincides with the disappearance of the double domain type wetting layer. LEED measurements invariably show a relaxed wetting layer with a single inplane lattice constant of $0.350 \mathrm{~nm}$ after the decay of the mesas. It thus appears that the disappearance of the double domain structure of the wetting layer destabilizes the earlier mentioned lock-in of the large $\mathrm{Pb}$ mesas at anchor sites related to the domain walls.

In conclusion, we have found that electronic QSE's are responsible for the initial shape of the rough surface of the lead films grown on $\mathrm{Pb}(111)$. The delicate balance between surface energies, elastic energies and QSE's is initially tilted towards QSE's, as discrete layer heights are observed. When the character of the film gradually changes from 2D to 3D, the balance between these forces becomes more and more dominated by interfacial energies, until it reaches a tipping point. The sudden decay of the electronically stabilized mesas is indeed just that, as the energetic balance is tipped for good in favor of the surface free energy, towards a compact structure. The spectacular speed at which the transition takes place is many orders of magnitude larger than what is expected, based on arguments involving thermally activated behavior. With a widespread interest in nanostructures in general, our results illustrate the generic need to characterize all aspects of nanostructures, both structural and electronic, since small excursions away from equilibrium can have dramatic consequences.

This work is part of the research programme of the Foundation for Fundamental Research on Matter (FOM), which is financially supported by the Netherlands Organisation for Scientific Research (NWO).

[1] L. Vitos, A. Ruban, H. Skriver, and J. Kollár, Surf. Sci. 411, 186 (1998).

[2] I. Markov, Crystal Growth for Beginners (World Scientific, Singapore, 2003).

[3] A. Krupski and S. Mróz, Surf. Rev. Lett. 10, 843 (2003).

[4] Z. Zhang, Q. Niu, and C.-K. Shih, Phys. Rev. Lett. 80, 5381 (1998)

[5] K. Budde, E. Abram, V. Yeh, and M. C. Tringides, Phys. Rev. B 61, R10 602 (2000). 
[6] M. Hupalo and M. C. Tringides, Phys. Rev. B 65, 115406 (2002).

[7] M. M. Özer, C.-Z. Wang, Z. Zhang, and H. H. Weitering, J. Low Temp. Phys. 157, 221 (2009), .

[8] K. L. Man, M.C. Tringides, M.M.T. Loy, and M.S. Altman, Phys. Rev. Lett. 101, 226102 (2008).

[9] M. Hupalo and M. C. Tringides, Phys. Rev. B 75, 235443 (2007).

[10] L. Kuipers and J. W. M. Frenken, Phys. Rev. Lett. 70, 3907 (1993).

[11] M. Nowicki, C. Bombis, A. Emundts, and H. P. Bonzel, Phys. Rev. B 67, 075405 (2003).

[12] K. Umezawa, S. Nakanishi, and T. Yumura et al., Phys. Rev. B 56, 10585 (1997).

[13] B. W. Dodson, Phys. Rev. Lett. 60, 2288 (1988).

[14] V. I. Marchenko, JETP Lett. 33, 381 (1981).

[15] R. van Gastel, R. Plass, N. C. Bartelt, and G. L. Kellogg, Phys. Rev. Lett. 91, 055503 (2003).
[16] See Supplemental Material at http://link.aps.org/ supplemental/10.1103/PhysRevLett.107.136103 for an example of shape transitions from unstable 3 layers high $\mathrm{Pb}$ islands into stable 5 layers high ones at $422 \mathrm{~K}$.

[17] H. Brune, K. Bromann, H. Röder, K. Kern, J. Jacobsen, P. Stoltze, K. Jacobsen, and J. Nørskov, Phys. Rev. B 52, R14380 (1995).

[18] B. Poelsema, J.B. Hannon, N.C. Bartelt, and G. L. Kellogg, Appl. Phys. Lett. 84, 2551 (2004).

[19] J. Heyraud and J. Métois, Surf. Sci. 128, 334 (1983).

[20] S. Surnev, K. Arenhold, P. Coenen, B. Voigtländer, H. Bonzel, and P. Wynblatt, J. Vac. Sci. Technol. A 16, 1059 (1998).

[21] M. Sano, T. Adaniya, T. Fujitani, and J. Nakamura, J. Phys. Chem. B 106, 7627 (2002).

[22] S. M. Binz, M. Hupalo, and M. C. Tringides, Phys. Rev. B 78, 193407 (2008). 